\title{
PARAMETRIC MODELLING FOR BETTER BRA FIT \& DESIGN
}

\author{
Ariya "Хихи" Amoozegar-Montero, School of Architecture and Design, Victoria University of \\ Wellington, New Zealand, amoozeariy@myvuw.ac.nz
}

Edgar Rodriguez Ramirez, School of Architecture and Design, Victoria University of Wellington, New Zealand,edgar.rodriguez-ramirez@vuw.ac.nz

\begin{abstract}
This paper presents the necessary parameters and measurements criteria that are critical for designing and modeling bra structure for an individual fit. The essential body data is collected using 3D scanning to form a digital model. This model is then used as an input of a parametric modelling of a semiautomated process to landmark, and draws the necessary measurements. This process allows for an efficient process that could ultimately allow for individually designed bras that would provide a better and more tailored fit.
\end{abstract}

\section{BACKGROUND}

It has been more than a hundred years since Mary Phelps Jacob received a patent for the first bra design that is commonly recognized as the basis for modern bras. And about the same time, the First World War accelerated the role of women in society, putting many women to work in factories and uniforms for the first time. The war may have also helped influence social mores, freeing women from constraining and uncomfortable corsets. On the other hand, for generations after that, mainstream underwear brands have infiltrated society's perception of the ideal body image. From the 'teardrop' silhouette bra of the 70 s to the rounded and lifted bra shape of the 80 s, salient advertisement campaigns have shaped perceptions of normality and desirability, promoting a narrow conceptualization of beauty and femininity. However, in the recent years there has been a movement to increase female body positivity. Many brands feature a celebration of the natural female form, incorporating a diverse range body types to transcend normative understanding of the 'perfect' shape and size. It has been suggested that at least $70 \%$ of women wear the incorrect bra size, with large breasted women accounting for the majority of this discrepancy [1-3]. The fundamental elements of the current bra fitting system incorporate overbust and underbust size [4]. However, these factors are insufficient for a proper fit. Though some women may have similar band and cup size, any particular bra will not fit these women exactly the same way. Research suggests that the smallest variations in body measurements can cause a significant difference, specifically up to a three size differential in the standard bra sizing system [3]. Therefore, reliance on the current bra sizing system is left wanting and inadequate for a "perfect" bra fit. This paper, as part of a larger research [5], addresses one of the important components of advanced bra design - a parametric modeling technique which would allowed for individually designed bra to increase a better bra fit for comfort, and support without compromising aesthetic.

\section{INTRODUCTION}

It is a common complaint by women about limitations of size and lack of proper standardization in bra selection. The current common A through E "standard" for cup size has partitioned the women's breasts into five discrete categories, implying over 3 billion women around the world can fit to these rather arbitrary sizes, when in reality the left and right breast on a single woman may not even be of the same 
size, or shape. It is essential to understand this gap between women's needs in terms of fit, comfort and esthetic and what is offered in the market. If cost is not an issue then each woman could have a personalized designed and fitted bra, but for a mass consumption of goods (i.e., affordable) a use of advanced technology and better design are needed to manufacture a product that can be used by masses while it is personalized. Current bra fitting techniques do not consider appropriate breast characteristics to improve bra fit, which can lead to discomfort, pain and even health issues. It has been shown to achieve an accurate fit of a bra detailed measurements of the breasts, and breast geometry, are necessary. The research has led to the design of a bra fitting and design system that allows each user to be measured for her unique contour and individual body shape. Due to the symbiotic relationship between bra fit and bra design, the project's system has been designed parametrically in order to digitize the measuring process as well as offer bra wearers an opportunity to provide preferences for bra fit and design.

Currently there are two possible options for breast measurement procedures in the bra fit system. The first is measuring breasts in the manufacturing stages, as part of bra design, patternmaking, and grading development. The second is to measure the breasts in the retail setting when purchasing a bra, used for fitting into the correct bra size of the individual wearer. If the goal is to design a better fitting bra, there must be recognition and a better understanding of the structure, support and function bras must provide. Ultimately, the design of bras cannot be independent of the characteristics of the woman's body and breasts. Very little research has been conducted on the bra design process [6].

Over last couple decades, computer-aided design (CAD) systems have been used for pattern amendment and grading to establish a more productive and efficient way to design bras. CAD systems are being used for shortening the product development cycle by improving pattern accuracy and grading sampling [7]. CAD offers an opportunity for more accurate approach to sizing and measuring as well as being able to draft a design on the computer before a physical form as such to reduce material waste. However, there is a clear need for updated CAD systems that offer an alternative way to create bra design forms not based on the manual pattern making approach. 3D scanning has been validated as an effective method for breast imaging. Unlike other breast imaging techniques, the 3D scanner has the ability to create accurate surface images of the complicated breast contour. The scanner is able to capture 3D breast models, which can later be analyzed to obtain the necessary measurements for bra design.

\section{METHODOLOGY}

In this research, 3D scanning technology was used to obtain accurate breast images of a number of volunteers. In case of 3D body scanning, there are several methods for measurement purposes: scanning the breasts in the nude state, scanning the desired breast shapes by asking the participant to lift her breasts to where she wants the bra to be fit, and finally, based on the softly supported breasts, asking the participant to wear a soft bra. In this research, nude figure method was selected. Lifting breasts option was eliminated because the determination of participant's desired breast position varies from one to another, and from time to time. Similarly, different soft bra styles create different breast shapes, and consequently wide variations. Nude breasts option, however, gives unique original data that can reflect the true size and natural shape of the breasts. Each participant was asked to stand in a relaxed position with their hands on their lower waist, while the lead author captured the breasts and upper torso data, using a hand-held scanner. Once scan data had been acquired, each scan was examined and any unnecessary imaging, such as the participant's partial image of head or arms, were removed from the scanned model (Figure 1). 

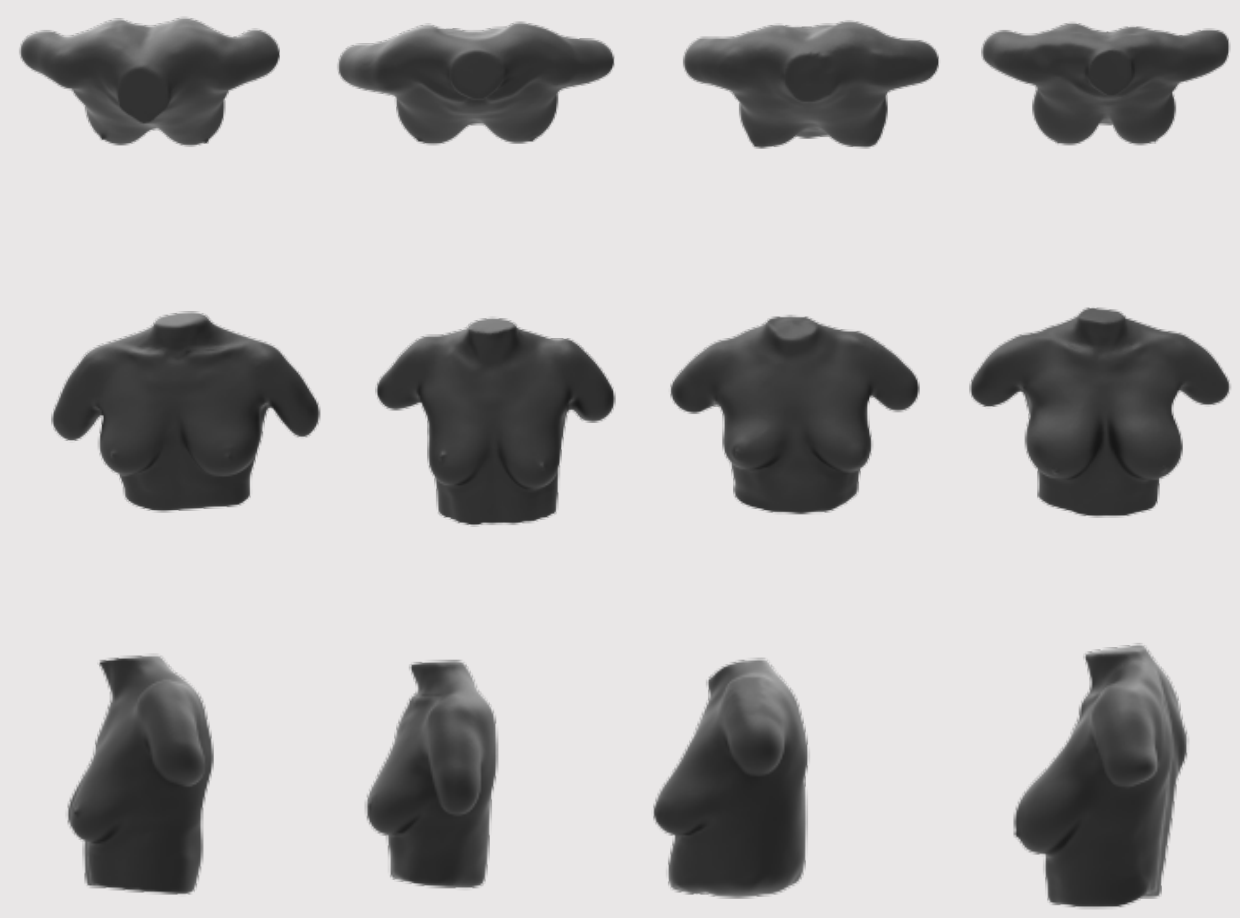

Figure 1: Sample of 3D scanned images

The parametric CAD software, Rhino Grasshopper, was used to extract the body data and appropriate measurements obtained from the 3D scanner. Through this software, the 3D model is manipulated and analyzed to assess accurate breast measurements needed specifically for the bra design process. Grasshopper is a parametric-based software within Rhino 3D system that allows the user to apply the same measuring processes for each 3D scanned model that is inputted into the program, instead of measuring manually each time. The software stores the designed formula needed to extract the measurements. The Rhino Grasshopper software was used to create a series of parametric "definitions" that will help standardize the process of identifying the necessary landmarking points ${ }^{1}$, as well as group these points in order to extract accurate breast measurements for better bra fit and individualized design (discussed further below, and displayed in Figure 3). Initially, six landmarking points are identified manually (e.g., Bottom Breast Point [BBP], a point along the inframammary fold of the breast) on the scan [8], which form a baseline. Additional points are automatically placed according to the designed Grasshopper definition list, which depends on the placement of the first six manual points. This insures that when a new scan is inputted into the system, the measurements will not need to be re-calculated, as long as the six landmarking points are placed correctly and consequently all other measurements should follow accurately as well (Figure 2). The Landmarks and anatomical reference points include: Upper breast point (UBP), upper breast point A (UBPA), center shoulder point (CSP), the bust point (BP), front armpit points (FAP), inner breast point (IBP), outer breast point (OBP), side waist point (SWP), the bottom breast point (BBP), front center point (FCP), and front neck point (FNP). These points help establish a clear understanding of how to reference the breast and are vital in developing accurate measurements and designing adequate bra fit. For example, the breast outline, which is the curve that

\footnotetext{
${ }^{1}$ Landmarking points are a series of points on the breasts that have been validated to extract accurate measurements of the breasts.
} 
defines the boundaries of the breast, is obtained through the landmarking points, OBP, BBP and IBP. The breast outline is a necessary measure to help design the underwire as well as the bottom bra cup shape. Though some points and measurements for a bra design will be static, many measurements will be highly variable and dependent on the bra style and specific design, such as the band or strap design. For example, the cup design depends on, the Inner Breast Point (IBP), Upper Breast Point A (UBPA), Outer Breast Point (OBP) and BBP, where the IBP and UBPA positioning can be variable depending on what type of cup style is preferred by the bra wearer. Sliders are designed in the Grasshopper definition to control the movement of these variable points in order to allow for easier adjustments and userfriendly designing. In other words if the designer would like to change a part of the design, e.g. the back band region, of the bra, he/she would choose the slider that controls that specific area of the band design. All the other points will adjust accordingly to make sure that the bra design always adapts back to the 3D scanned model.
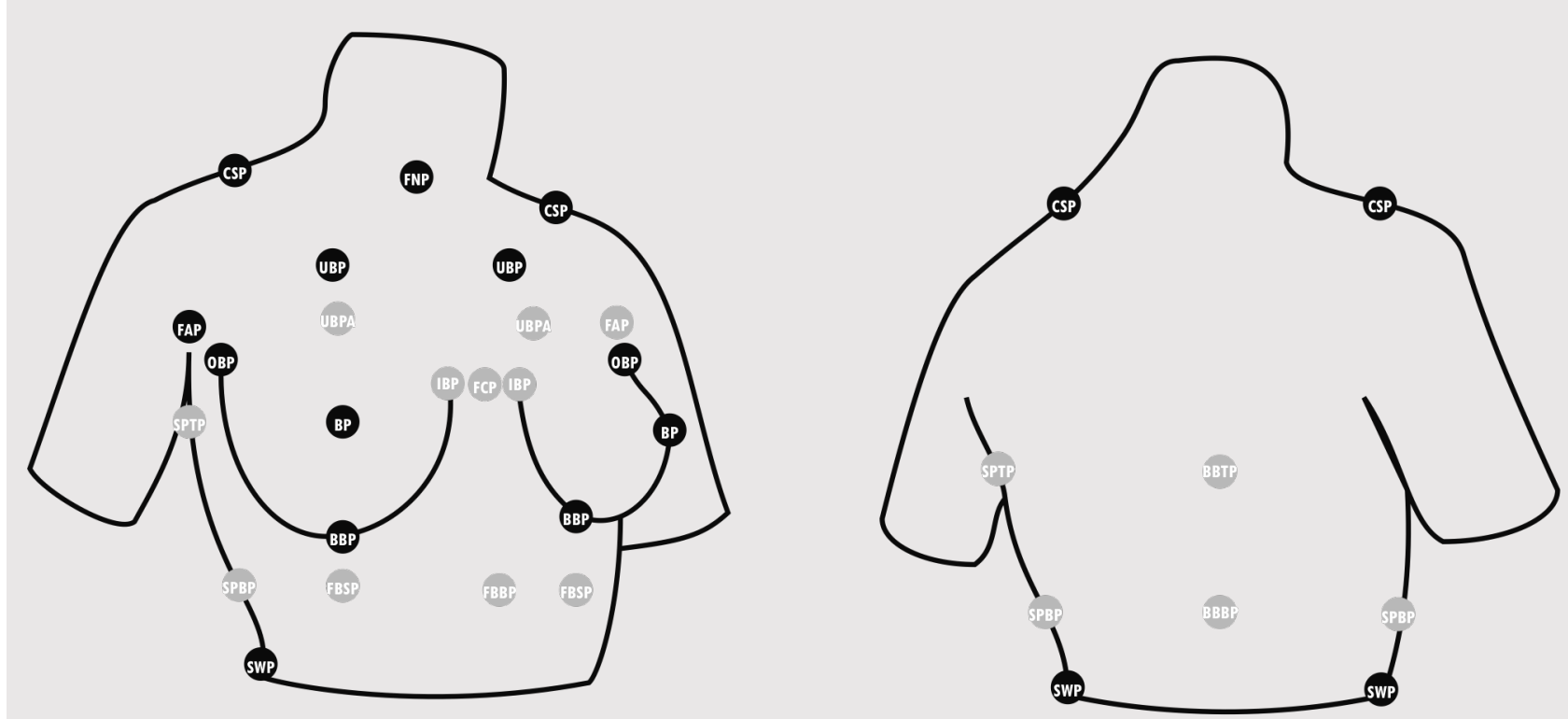

Figure 2: Static landmarking points in black, and variable landmarking points in grey

Figure 3 displays modelling in the Rhino Grasshopper software to utilize the input, the 3D scanned model mesh (point A), and set the manual landmarking points that are then used to develop the additional landmarking points automatically and corresponding breast measurements needed for individualized fitted bra design. For example, the top right box (point B) contains a set of definitions that set the automated landmarking point (UBPA) and determine the measurements needed for the right breast for the right bra cup design (BP to BBP; $\mathrm{BP}$ to IBP, BP to OBP; IBP to BBP to OBP; IBP to UBPA to OBP) based upon the selected relevant manual points (BP, IBP, and BBP) and the relevant automated point found in the box on the top left (OBP) (point C). Highlighted in bright blue is the process of obtaining the Outer Breast Points (OBP) parametrically through the programming of a specific definition sequence to create this formula. The OBP can be calculated through the midpoint of the Front Armpit Point (FAP) and the intersection of FAP and the Side Waist Point (SWP), and the 
horizontal line passing through the BBP [8]. So the OBP is unique to the position of the BBP, FAP and SWP of both right and left breast, and thus must be considered be evaluated separately.
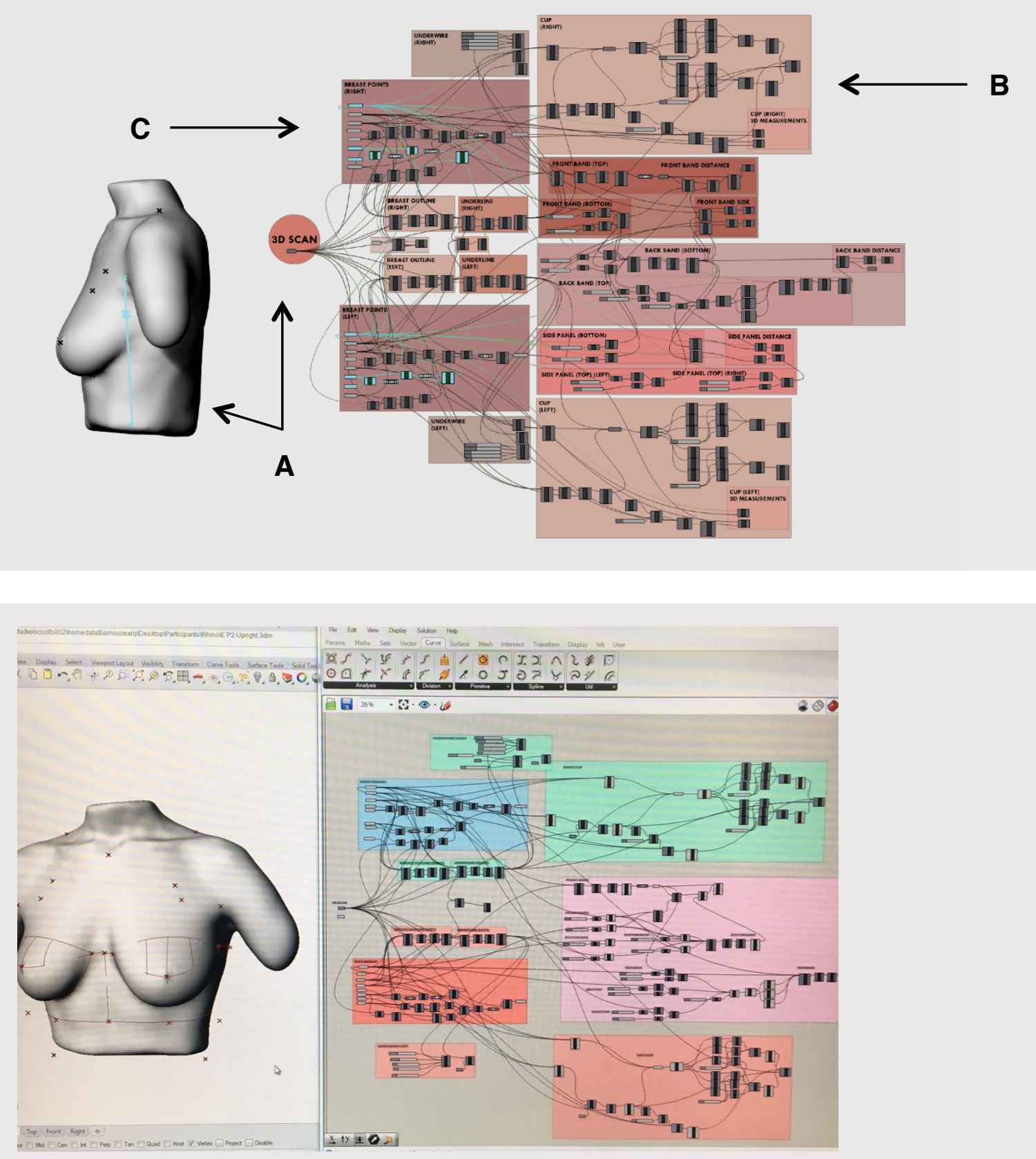

Figure 3: Automated landmarking of the OBP; Grasshopper to bra design

Once the landmarking points have been established, an analyzing technique could be used to compare the breast scans. Participants, who self-identified their bra size as 12 D (NZ size), were select for the research. It should be noted however each participant explained that the bras they fit into were actually within a wide range of sizes. This meant though every participant, at some point, was wearing the same 
size as indicated on the label of the bra, they were also wearing a range of sizes that were different from each other.

Using the landmarking technique described above, we were able to draw outlines of the different contours of the breast shape in order to further examine these variances. The first set of sketches outlined the points OBP to BBP to IBP for each breast, where a line is drawn through these points to create an underbust line, a line that is used to measure and design the underwire shape. The points between the two IBP are also connected in this sketch to outline the front band distance. The second set looks at the points drawn from OBP to BP to IBP for each breast. This can be understood at the bust point line. When the first set and second set are combined and viewed from a perspective angle it becomes quite interesting. Here, the differences between bust heights are examined. One can notice that each participant has a different bust point (BP) altitude and angle. Moreover, for each participant there are different BP heights between the right and left breast. Figure 4 illustrates these points, using sketches for each participant.

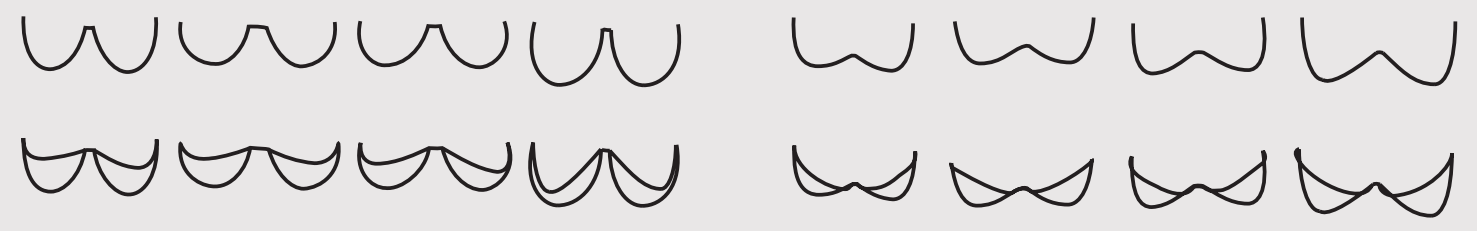

Figure 4: Sketch of landmarking points utilized for underline and bust line comparisons

\section{CONCLUDING REMARKS}

There are many applications to the process proposed in this research. Most bra design and manufacturing companies are seeking better ways of making bras that are aesthetically pleasing, are affordable and meet the changing demand (as well as body shape and contour) of their customers. The gap between what the users of bra need and require and what the bra manufacturers (and designers) provide is wide. And although this paper and its associated larger research by itself cannot revolutionized the process or even the culture of bra designing, manufacturing in one side and bra fitting and selection on the other, it has opened new avenues of exploration for developing a process and concept for this important garment.

In this paper, we focused on the digital parametric measuring system based on landmarking points and corresponding measurements based on an individual 3D scanned digital mesh model of the person. This system allows for the necessary measurements to adapt to the individual 3D scan that is uploaded, and then store all data digitally so that the exact measurements can be referenced at a later point. This is adaptive measurement is an essential part of manufacturing system that needs to mass produce a product (to keep the price relatively low), while attempting to respond to individual body types and consumers' sense of fit and comfort. We invite the interested readers to see lead author's work [1] for more details of using the parametric modelling for advanced manufacturing of individually designed and fitted bras. 
In the current market, mass produced bras (or essentially any product) are designed for a generic body types with discrete size steps. This was a fine response to growth in population and demand for availability of product for masses. In the past, as in the present, the very wealth can afford to have individually designed clothing or undergarment. The advent of 3D scanning, 3D knitting, 3D printing and other advanced manufacturing technologies have allowed for a democratisation consumer product. As discussed in this research, many of these products are not sensitive to size fluctuation. However, in bra design and manufacturing, small fluctuation in size and fit can have a dire impact on comfort. Hence, the importance of this researcher's contribution in adaptive measurement, gaps in measurements and human factor.

\section{REFERENCES}

1. McGhee, D. E., \& Steele, J. R. Optimising breast support in female patients through correct bra fit. Journal of Science and Medicine in Sport, 13, 568-572, 2010.

2. Tsarenko, Y., \& Strizhakova, Y. Insights into Gendered Consumption: Modeling Retailer Outcomes and Consumer Shopping Characteristics. Developments in Marketing Science: Proceedings of the Academy of Marketing Science Marketing in Transition: Scarcity, Globalism, \& Sustainability, 157-160, 2015.

3. White, J., \& Scurr, J. Evaluation of professional bra fitting criteria for bra selection and fitting in the UK. Ergonomics, 55(6), 704-711, 2012.

4. McGhee, D. E. Sports bra design and bra fit: Minimising exercise-induced breast discomfort. (Doctoral Thesis, University of Wollongong, Wollongong, Australia), 2009.

5. Amoozegar-Montero, Ariya, Parametric Modelling and Digital Manufacturing for Better Bra Fit, Masters Thesis, Victoria University of Wellington, 2016.

6. Hardaker, C., \& Fozzard, G. The bra design process: a study of professional practice. International Journal of Clothing Science and Technology Int Jnl of Clothing Sci \& Tech, 9(4), 311-325, 1997.

7. Yip, J., \& Yu, W. Intimate apparel with special functions. Innovation and Technology of Women's Intimate Apparel, 171-195, 2006.

8. Lee, H., K. Hong and E. Kim, Measurement Protocol of Women's Nude Breasts Using a 3D Scanning Technique, Applied Ergonomics, Volume 3 35, 2004. 